\title{
LA REGULACIÓN DE LOS ENSAYOS CLÍNICOS PARA LA COVID-19 EN EL PERÚ
}

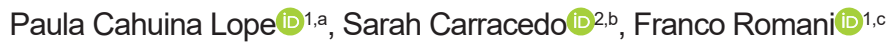 \\ 1 Oficina General de Investigación y Transferencia Tecnológica, Instituto Nacional de Salud, Lima, Perú \\ 2 Pontificia Universidad Católica del Perú, Lima, Perú \\ a Médico Cirujano, Especialista en Medicina Familiar y Comunitaria; ${ }^{b}$ abogada, magíster en Bioética; ${ }^{\mathrm{c}}$ médico cirujano, \\ magister en Epidemiología
}

\section{RESUMEN}

La COVID-19 ha afectado la conducción de los ensayos clínicos a nivel mundial. Iniciada la emergencia sanitaria en Perú, el gobierno nacional tomó medidas para promover y garantizar la realización de los ensayos clínicos en COVID-19. Se conformó un comité de ética nacional exclusivo para ensayos clínicos COVID-19 y se implementó una regulación para asegurar la realización ética y oportuna de ensayos clínicos. Hasta el 31 de diciembre del 2020, el Instituto Nacional de Salud autorizó 29 ensayos clínicos, de los cuales cinco evalúan vacunas. El tiempo promedio y desviación estándar de la autorización fue 19,3 y 10,5 días, respectivamente. El 58,6\% ( $n=17)$ fueron ensayos clínicos fase II y el 34,5\% $(n=10)$ fueron fase III; el 31,0\% (n=9) tuvo como patrocinador a una institución peruana. La finalidad de las acciones implementadas fue promover la investigación para la COVID-19, respondiendo a las necesidades y tiempos de la emergencia sanitaria, sin afectar la protección de los participantes ni la rigurosidad de los estudios.

Palabras clave: COVID-19; Ensayos Clínicos como Asunto; Infecciones por Coronavirus; Perú; Regulación Gubernamental. (Fuente: DeCS BIREME).

\section{THE REGULATION OF COVID-19 CLINICAL TRIALS IN PERU}

\begin{abstract}
COVID-19 has affected the conduct of clinical trials worldwide. Once the health emergency began in Peru, the national government took measures to guarantee the conduct of COVID-19 clinical trials. A national research ethics committee was exclusively established for COVID-19 clinical trials; and a regulatory framework was implemented to ensure the ethical and timely conduct of these studies. To december 31, 2020, the Peruvian National Health Institute authorized 29 clinical trials, of which 4 test vaccines. The mean and standard deviation of time authorization were 19.3 and 10.5 days, respectively. $58.6 \%(n=17)$ were phase II clinical trials and $34.5 \%(n=10)$ were phase III; $31.0 \%(n=9)$ were sponsored by a Peruvian institution. The aim of the actions implemented was to promote COVID-19 research while responding to the health emergency needs without affecting the protection of participants or the rigor of the studies.
\end{abstract}

Keywords: COVID-19; Clinical Trials as Topic; Coronavirus Infections; Peru; Government Regulation. (Source: MeSH NLM).

\section{INTRODUCCIÓN}

Citar como: Cahuina-Lope P, Carracedo $\mathrm{S}$, Romaní $\mathrm{F}$. La regulación de los ensayos clínicos para la COVID-19 en el Perú. Peru Med Exp Salud Publica. 2021;38(1):171-7. doi: https://doi. org/10.17843/rpmesp.2021.381.6627.

Correspondencia: Paula Cahuina Lope; pcahuina@ins.gob.pe

Recibido: $26 / 10 / 2020$ Aprobado: 03/02/202 En línea: 10/02/2021
Frente a la actual crisis sanitaria por la pandemia de la enfermedad por el coronavirus (COVID-19), la humanidad requiere de la rápida búsqueda e introducción de intervenciones sanitarias orientadas a combatir la COVID-19. La respuesta de los científicos a nivel mundial ha sido sorprendente; entre diciembre del 2019 y mayo del 2020, 7440 artículos sobre COVID-19 fueron publicados ${ }^{(1)}$. Para enero del 2021 se han registrado 68760 artículos originales en la plataforma COVID-19 Open Research Dataset $^{(2)}$. Algunos de estos resultados de investigación corresponden a estudios que han formulado y evaluado diversos candidatos de medicamentos y vacunas para la COVID-19, incluyendo ensayos clínicos (EC). Los EC constituyen la piedra angular para generar evidencia necesaria sobre la eficacia y seguridad de los productos en investigación, y son requeridos por las instancias reguladoras para la autorización de nuevas intervenciones de salud ${ }^{(3)}$. 
A nivel mundial, los EC deben adherirse a estándares de ética en investigación, así como cumplir con las buenas prácticas clínicas y los procesos regulatorios de las autoridades sanitarias con la finalidad de proteger los derechos, la seguridad y el bienestar de los participantes ${ }^{(4)}$. Sin embargo, la pandemia ha desafiado los procesos ordinarios de autorización y conducción de los EC pues, por un lado, los EC activos tienen problemas para su ejecución debido a las restricciones de un estado de emergencia sanitaria - por ejemplo, el acceso a los centros de investigación por parte de los participantes ha sido imposibilitada por las medidas de inmovilización social—. Por otro, los EC de COVID-19 han requerido plazos cortos y procesos flexibles para su aprobación ética y autorización regulatoria ${ }^{(4,5)}$.

En el Perú se ha adecuado el marco regulatorio para que responda a los desafíos que una emergencia sanitaria plantea, sin sacrificar las exigencias que garantizan la realización ética y oportuna de los EC. El objetivo de este artículo es describir estas adecuaciones en la regulación nacional de los EC para la COVID-19, así como describir las características principales de estos estudios en el Perú hasta diciembre del 2020.

\section{EL MARCO REGULATORIO DE LOS ENSAYOS CLÍNICOS DE COVID-19 DURANTE LA PANDEMIA}

La investigación científica durante la pandemia es un deber ético, pues sus resultados permiten mejorar la atención y manejo clínico de las personas con COVID-19 ${ }^{(6)}$. No obstante, llevar a cabo investigaciones durante una emergencia sanitaria es un proceso complejo pues se requiere generar resultados en periodos cortos de tiempo, sin afectar la rigurosidad de los estudios, ni los aspectos éticos que rigen toda la investigación con seres humanos. Al igual que en condiciones habituales, durante una emergencia sanitaria las propuestas de investigación con seres humanos deben ser revisadas y aprobadas por un comité de ética en investigación antes de su inicio. Evidentemente, esta obligación incluye a los EC ${ }^{(7)}$.

La urgencia de soluciones para hacer frente a la COVID-19 no es justificación para que los investigadores no se adhieran a los estándares nacionales e internacionales de ética en investigación. A pesar de ello, los procedimientos habituales de revisión de los comités de ética en investigación y los tiempos que estos manejan no suelen ser los adecuados para una situación de desastre o brote epidémico ${ }^{(8,9)}$. Por ende, es necesario implementar procesos de revisión y supervisión éticas rápidos, flexibles y rigurosos durante la pandemia ${ }^{(10)}$.

El 12 de abril de 2020 se publicó un decreto supremo cuya finalidad es promover y garantizar la realización ética y oportuna de los ensayos clínicos en COVID-19 durante la emergencia sanitaria. Dicha norma dispone la creación del Comité Nacional Transitorio de Ética en Investigación para la evaluación y supervisión ética de los Ensayos Clínicos de la enfermedad COVID-19 (CNTEI-COVID-19); así como la elaboración de procedimientos para la autorización de EC en COVID-19.

La estrategia elegida corresponde a la creación de un comité de ética en investigación ad-hoc e independiente ${ }^{(8)}$, integrado por diversos miembros de Comités Institucionales de Ética en Investigación acreditados ante el Instituto Nacional de Salud (INS) ${ }^{(11)}$. El CNTEI-COVID-19 tiene la competencia de revisar los protocolos de investigación y supervisar los ensayos clínicos con productos farmacéuticos y dispositivos médicos para la prevención, diagnóstico y tratamiento de la COVID-19 que se lleven a cabo en el Perú durante la emergencia sanitaria. Su finalidad es proteger los derechos, bienestar y seguridad de los sujetos de investigación ${ }^{(12)}$. A diferencia de otros comités de ética de la investigación, el CNTEI-COVID-19, además de su exclusividad en materia de COVID-19, se caracteriza por sus procedimientos flexibles para la recepción de protocolos de investigación, tiempos cortos para la revisión ética, el uso de medios electrónicos para las sesiones y comunicaciones con el patrocinador, y la exoneración de pagos en los trámites (Tabla 1).

La creación del CNTEI-COVID-19 no fue la única estrategia para agilizar la conducción de EC durante la pandemia. Los procedimientos del INS - entidad regulatoria de EC en el Perú - también se adecuaron para promover la oportuna ejecución de EC durante la emergencia sanitaria. Por ello, la Oficina General de Investigación y Transferencia Tecnológica (OGITT) del INS, implementó un procedimiento especial para la autorización de $\mathrm{EC}^{(11,12)}$. Los aspectos más relevantes de dicho procedimiento son los siguientes: la reducción del número de requisitos de 20 a 10; la reducción de los tiempos de autorización de 30 a 7 días hábiles; la posibilidad de que el patrocinador pueda solicitar directamente a la Autoridad Nacional de Medicamentos (ANM) la opinión técnica sobre el perfil de seguridad y calidad del producto en investigación; la utilización de una mesa de partes virtual, el uso de medios electrónicos, reuniones virtuales y la vía telefónica para la comunicación con el patrocinador; así como la exoneración de pagos ante el INS.

Con las estrategias empleadas, hasta el 31 de diciembre del 2020, el promedio del tiempo para la autorización fue de 19,3 días con una desviación estándar (DE) de 10,5; y un rango de 3 a 39 días. Además, la mitad de los EC fueron autorizados entre 10 a 26 días. Estos tiempos tienen dos componentes: el atribuido al INS, que incluye el plazo para revisar la documentación, analizar los datos, formular los informes técnicos, hasta la emisión de la Resolución Directoral que autoriza o no el EC; y el tiempo atribuido al patrocinador para responder a las observaciones planteadas por el INS. El promedio del tiempo regulatorio atribuido del INS fue de 4,2 días (DE: 2,7) con un rango de 1 a 12 días. Solo en una de las 29 solicitudes el tiempo excedió los 7 días. Los productos de investigación de origen químico tuvieron una media del tiempo regulatorio global de 19,1 días (DE: 12,4; 
Tabla 1. Comparación entre los Comités Institucionales de Ética de la Investigación acreditados y el CNTEI-COVID19

\begin{tabular}{|c|c|c|}
\hline Característica & $\begin{array}{l}\text { Comités Institucionales de Ética de la Investigación } \\
\text { (CIEI) }\end{array}$ & CNTEI-COVID19 \\
\hline Alcance & Nivel institucional & Nivel nacional \\
\hline Finalidad & \multicolumn{2}{|c|}{ Proteger los derechos, bienestar y seguridad de las personas que participan en los ensayos clínicos. } \\
\hline Competencia & $\begin{array}{l}\text { Revisar y supervisar todos los ensayos clínicos con } \\
\text { productos farmacéuticos y dispositivos médicos que se } \\
\text { realizan en su institución o con involucramiento de su } \\
\text { institución. Algunos CIEI revisan y supervisan ensayos } \\
\text { clínicos de terceras instituciones siempre y cuando ha- } \\
\text { yan sido designados para ello. }\end{array}$ & $\begin{array}{l}\text { Revisar y supervisar todos los ensayos clínicos con pro- } \\
\text { ductos farmacéuticos y dispositivos médicos de la enfer- } \\
\text { medad COVID-19 que se realizan en todo el Perú, inde- } \\
\text { pendiente de las instituciones involucradas. }\end{array}$ \\
\hline Membresía & Mínimo 5 miembros titulares. & Mínimo 7 miembros titulares. \\
\hline
\end{tabular}

Deben incluirse miembros alternos.

Hombres y mujeres.

Presentación de protocolos Presentación física del expediente.

de investigación

Tiempo de revisión

Sesiones

Toma de decisiones

Base ética de las decisiones

Actas

Canales de comunicación

Supervisión de EC nidades.
El expediente debe cumplir con todos los requisitos para que los miembros inicien la revisión.

Depende de cada CIEI y de las observaciones al protocolo, pero en total los tiempos varían entre 1 y 3 meses.

Presenciales

Programación semanal, quincenal o mensual.

Suele ser la mitad +1 de los miembros, incluyéndose la presencia de todas los integrantes con las pericias requeridas.

Votan los miembros presentes en la sesión.
Profesional de la salud con conocimientos en metodología de la investigación, profesional médico con especialidad relacionada con la COVID-19, químico farmacéutico, profesional del derecho, profesional de las ciencias conductuales o sociales, profesional con formación en bioética y representante de la comunidad.

Presentación virtual del expediente.

El expediente puede estar incompleto para que los miembros inicien la revisión (presentación flexible de requisitos).

Depende de las observaciones, pero los miembros tienen 3 días calendario para revisar un protocolo desde que es recibido.

Virtuales

Programación varía según los protocolos de investigación. Las sesiones se programan, a más tardar, a los 5 días calendario de presentado un protocolo de investigación.

Mitad +1 de los miembros titulares.

Votan los miembros presentes en la sesión y aquellos que no puedan asistir pero que hayan enviado sus revisiones y decisiones con antelación (participación diferida).

Consenso, o en su defecto, por mayoría simple.

1) Validez científica, 2) Valor social, 3) Selección equitativa de los participantes, 4) Balance riesgos-beneficios, 5) Proceso de consentimiento informado, 6) Respeto por las personas, 7) Participación e involucramiento de las comu-

Actas físicas, suscritas a mano.

A través de cartas u oficios, por medio de las autoridades institucionales.

Se solicitan informes de avance, reportes de EAS, desviaciones, entre otros. Supervisiones in situ.
Actas virtuales, suscritas electrónicamente.

A través de medios electrónicos, por medio del presidente del CNTEI-COVID19.

Solicitud de informes de avance mensuales, reportes de EAS, desviaciones, entre otros. Supervisiones in situ o virtuales según el contexto de la emergencia. Seguimiento ético constante sincrónicos o diferidos. 
rango 3 a 28), los biológicos tuvieron una media de 19,0 días (DE: 11,6; rango 4 a 39), los hemocomponentes 20,3 días (DE: 3,9; rango de 18 a 26). Solo se autorizó un producto dietético con un tiempo global de 26 días.

Otra adecuación en el trámite de autorización fue permitir la simultaneidad en la revisión y aprobación ética del CNTEI-COVID-19, la solicitud de opinión del perfil de seguridad y calidad del producto ante la ANM, y la solicitud de autorización del EC ante el INS. La simultaneidad de estos procedimientos contribuye a disminuir los tiempos de revisión y promover la coordinación entre las instancias involucradas. Sin embargo, como en situaciones ordinarias la naturaleza vinculante entres dichas decisiones permanece. Es decir, si el CNTEI-COVID19 no aprueba un protocolo de EC o si la ANM no da su opinión favorable, el estudio no es autorizado por el INS ${ }^{(13,14)}$.

La adecuación en el seguimiento y supervisión a los ensayos clínicos autorizados ha sido implementada por el CNTEI-COVID-19 y el INS. El CNTEI-COVID19 cuenta con mecanismos virtuales y electrónicos de supervisión ética; y solicita mensualmente a los investigadores información sobre el progreso de sus EC. Por su parte, hasta el 31 de diciembre del 2020, el INS ha realizado inspecciones presenciales a 11 EC en COVID-19 de los 29 autorizados, es decir al 37,9\%. Dicha proporción es superior al porcentaje de EC inspeccionados entre el total de EC activos, que en el año 2018 fue del 17,1\%; en el 2019, 11,5\% y en el 2020 fue del 10,5\% para EC no COVID-19.

\section{CARACTERÍSTICAS DE LOS ENSAYOS CLIINICOS DE COVID-19 EN EL PERÚ}

Desde la declaración de la emergencia sanitaria en el Perú, la comunidad científica peruana se ha comprometido en la generación de soluciones que contribuyan a la lucha contra la pandemia. A pesar de las dificultades y los retos que la emergencia sanitaria plantea en nuestro país, varios EC para encontrar tratamientos (incluyendo terapias profilácticas) y vacunas para la COVID-19 han sido autorizados.

El Registro Peruano de Ensayos Clínicos (REPEC) es una base de datos de acceso público, disponible en la página web del INS (https://ensayosclinicos-repec.ins.gob.pe) y tiene como objetivo garantizar la transparencia de los EC mediante la publicación de información básica sobre los EC autorizados por el INS ${ }^{(15)}$. El REPEC es un registro primario de la Plataforma de Registros Internacionales de Ensayos Clínicos de la Organización Mundial de la Salud (OMS) (ICTRP, por su sigla en inglés) desde el 2016.

De 1 de enero al 31 de diciembre del 2020, 96 EC fueron registrados en el REPEC. De estos, 29 (30,2\%) EC fueron en COVID-19, 22 EC evalúan productos de investigación para el tratamiento de esta enfermedad, 7 evalúan productos para su prevención de los cuales 5 son EC de vacunas. Solo un EC de carácter global se está llevando a cabo en Perú (el Ensayo Solidaridad de la OMS). Los EC fase II (58,6\%) y fase III $(34,5 \%)$ son lo más frecuentes. Existe un predominio de EC doble ciego $(65,5 \%)$ y aleatorizados $(93,1 \%)$. Todos los EC enrolan a mayores de 18 años, el 41,4\% de EC tiene planeado enrolar más de 100 participantes peruanos. Los criterios de valoración son variados, destacando el requerimiento de cuidados intensivos, la necesidad de ventilación mecánica y la mortalidad (Tabla 2).

Seis de 24 productos en investigación cuentan con registro sanitario en el Perú y tienen, según la Dirección General de Medicamentos Insumos y Drogas (DIGEMID), indicaciones para el tratamiento de otras enfermedades (Tabla 3). Los tipos de productos en investigación con más EC autorizados son el plasma convaleciente (4 EC) y las vacunas (5 EC). Entre las vacunas en estudio están la vacuna inactivada contra el SARS-CoV-2 de Sinopharm, la vacuna de ARNm mensajero de CureVac AG., la vacuna de vector viral no replicante basada en un adenovirus de chimpancé que expresa la proteína espiga del SARS-CoV-2 fabricada por Astrazeneca y la Universidad de Oxford, y otra vacuna de vector viral recombinante en adenovirus humanos que expresa la proteína espiga de la empresa Janssen ${ }^{(16)}$.

\section{DISCUSIÓN}

A nivel mundial, las entidades reguladoras de los EC, junto con los investigadores, empresas farmacéuticas e instituciones de investigación vienen jugando un rol crucial en el desarrollo de EC que buscan probar nuevas intervenciones diagnósticas, preventivas y terapéuticas para la COVID-19, para así obtener conocimiento valioso sobre su seguridad y eficacia. En este contexto, es crucial asegurar que los datos y resultados generados en los EC sean transparentes, interpretables, válidos y oportunos ${ }^{(17)}$, ya que de ello depende que estos productos obtengan un registro sanitario y puedan ser utilizados en la población que lo necesite.

El escenario actual plantea el reto de lograr un equilibrio entre la necesidad de generar soluciones rápidamente, proteger los derechos, seguridad y bienestar de los participantes de los estudios, y aplicar ciencia con altos estándares de calidad ${ }^{(18)}$. En atención a las recomendaciones de la OPS/ OMS y tal como ha ocurrido con agencias reguladoras de otras partes del mundo, como la Agencia Europea de Medicamentos (EMA) ${ }^{(19)}$, la Agencia Federal de Administración de Alimentos y Drogas (FDA, por sus siglas en inglés) de los Estados Unidos ${ }^{(20)}$, o la Agencia Nacional de Vigilancia Sanitaria (Anvisa) de Brasil ${ }^{(21)}$; en el Perú se han implementado medidas para la evaluación y autorización acelerada de EC ${ }^{(22)}$. Dichas medidas se adhieren a estándares éticos en investigación, garantizan la independencia de las instancias evaluadoras, y buscan que los plazos sean los apropiados para una situación de emergencia sanitaria. 
Tabla 2. Características generales de los ensayos clínicos en COVID-19 autorizados en Perú durante el 2020.

\begin{tabular}{|c|c|c|}
\hline Características del estudio & $\mathbf{n}$ & $\%$ \\
\hline \multicolumn{3}{|l|}{ Tipo de intervención } \\
\hline Preventiva & 7 & 24,1 \\
\hline Diagnóstica & 0 & 0,0 \\
\hline Terapéutica & 22 & 75,9 \\
\hline \multicolumn{3}{|l|}{ Tipo de producto en investigación } \\
\hline Químico & 11 & 37,9 \\
\hline Biológico & 12 & 41,4 \\
\hline Dietético & 1 & 3,4 \\
\hline Hemocomponente & 4 & 13,8 \\
\hline Mixto & 1 & 3,4 \\
\hline \multicolumn{3}{|l|}{ Tipo de Patrocinador } \\
\hline Extranjero & 19 & 65,5 \\
\hline Nacional & 9 & 31,0 \\
\hline Mixto & 1 & 3,4 \\
\hline \multicolumn{3}{|l|}{ Número de centros de investigación } \\
\hline 1 & 14 & 48,3 \\
\hline 2 & 3 & 10,3 \\
\hline$\geq 3$ & 12 & 41,4 \\
\hline \multicolumn{3}{|l|}{ Duración del ensayo clínico (meses) } \\
\hline 1 a 6 & 6 & 20,7 \\
\hline 7 a 12 & 14 & 48,3 \\
\hline 13 a 24 & 7 & 24,1 \\
\hline Más de 24 & 2 & 6,9 \\
\hline \multicolumn{3}{|l|}{ Fase Clínica } \\
\hline I-II & 1 & 3,4 \\
\hline II & 17 & 58,6 \\
\hline II/III & 0 & 0,0 \\
\hline III & 10 & 34,5 \\
\hline No aplica & 1 & 3,4 \\
\hline \multicolumn{3}{|l|}{ Tipo de cegamiento } \\
\hline Abierto & 8 & 27,6 \\
\hline Simple & 0 & 0,0 \\
\hline Doble & 19 & 65,5 \\
\hline Triple & 2 & 6,9 \\
\hline \multicolumn{3}{|l|}{ Aleatorización } \\
\hline No & 2 & 6,9 \\
\hline Sí & 27 & 93,1 \\
\hline \multicolumn{3}{|l|}{ Número de participantes } \\
\hline 1 a 100 & 17 & 58,6 \\
\hline 101 a 1000 & 8 & 27,6 \\
\hline$>1000$ & 4 & 13,8 \\
\hline \multicolumn{3}{|l|}{ Criterio de valoración principal } \\
\hline Efecto protector de la vacuna & 4 & 13,8 \\
\hline Mejora clínica & 3 & 10,3 \\
\hline Mortalidad & 5 & 17,2 \\
\hline $\begin{array}{l}\text { Participante vivo y sin insuficiencia respi- } \\
\text { ratoria }\end{array}$ & 4 & 13,8 \\
\hline Progresión de la enfermedad & 2 & 6,9 \\
\hline Prueba positiva para SARS-CoV-2 & 3 & 10,3 \\
\hline $\begin{array}{l}\text { Reactogenicidad y reportes de Eventos } \\
\text { adversos serios de la vacuna SARS-CoV-2 }\end{array}$ & 1 & 3,4 \\
\hline Reportes de eventos adversos & 1 & 3,4 \\
\hline $\begin{array}{l}\text { Requerimiento de cuidados intensivos, } \\
\text { ventilación y mortalidad }\end{array}$ & 6 & 20,7 \\
\hline Total & 29 & 100,0 \\
\hline
\end{tabular}

Las medidas implementadas han logrado resultados satisfactorios para los tiempos de revisión y aprobación éticas; así como en los plazos regulatorios para las autorizaciones de EC. En el caso del CNTEI-COVID-19, el compromiso, dedicación y responsabilidad de sus miembros explican su desempeño. En el caso del INS, la reducción de tiempos se debe principalmente a la calidad de recursos humanos asignados a la revisión de los EC, la priorización de la evaluación de los EC para COVID-19, los mecanismos de comunicación y coordinación rápida con los investigadores y patrocinadores y, en general, a la necesidad de cumplir con los plazos establecidos en el contexto de emergencia sanitaria por la COVID-19.

Como sucede en situaciones habituales, el promedio del tiempo de autorización de EC con productos químicos resulta menor a los EC con productos biológicos. En caso de los EC de plasma de donantes convalecientes de la COVID-19, el tiempo de autorización fue mayor puesto que antes de la pandemia, el INS no tenía competencias para los ensayos clínicos con hemocomponentes. No fue hasta la emergencia sanitaria que el Ministerio de Salud estableció que los EC de plasma convaleciente fueran autorizados por el INS, con la opinión de seguridad y calidad del Programa Nacional de Hemoterapia y Bancos de Sangre (PRONAHEBAS) ${ }^{(23)}$; lo que implicó una serie de nuevas coordinaciones y procedimientos entre las autoridades correspondientes.

Un aspecto resaltante es la proporción de patrocinadores nacionales de los EC en COVID-19, la cual se aproxima al 35\% siendo una clara muestra del rol protagónico que han tomado investigadores e instituciones de investigación nacionales para asumir el liderazgo y responsabilidad de conducir estos estudios en búsqueda de intervenciones que podrían resultar beneficiosas para la COVID-19. Este dato dista mucho del reportado en los EC autorizados en el Perú entre 1995 y 2012, donde en 8 (0,7\%) de 1255 EC autorizados hubo patrocinio por una institución peruana ${ }^{(24)}$.

El CNTEI-COVID-19 y el INS realizan un seguimiento cercano a la ejecución de los EC para la COVID-19; lo cual es fundamental para asegurar el bienestar de los participantes y velar por la calidad e integridad de los datos en un contexto caracterizado por la incertidumbre y la generación rápida de evidencia sobre ciertos productos en investigación. La importancia de esta vigilancia activa se ha evidenciado, por ejemplo, ante las rápidas acciones que se tomaron frente al surgimiento de evidencia científica que llevaron a la suspensión y luego cancelación de los brazos de hidroxicloroquina y lopinavir/ritonavir por demostrarse su futilidad en el ensayo clínico Solidaridad ${ }^{(25)}$ o ante los eventos adversos serios ocurridos con los estudios de vacunas de AstraZeneca-Universidad de Oxford ${ }^{(26)}$, y Janssen ${ }^{(24)}$.

En conclusión, la pandemia de la COVID-19 ha impactado la regulación de EC a nivel mundial, y Perú no ha 
Tabla 3. Productos en investigación de los ensayos clínicos COVID-19 autorizados en Perú

\begin{tabular}{|c|c|c|c|}
\hline Producto en investigación & Nùmero de EC & $\begin{array}{c}\text { Registro } \\
\text { sanitario }\end{array}$ & Indicaciones comunes $^{\mathrm{a}}$ \\
\hline $\mathrm{ABX} 464$ & 1 & No & Ninguna; producto en investigación. \\
\hline Acalabrutinib & 2 & No & Ninguna; producto en investigación. \\
\hline ACTIV-1 IM & 1 & No & Ninguna; producto en investigación. \\
\hline Antroquinolol & 1 & No & Ninguna; producto en investigación. \\
\hline DFV890 & 1 & No & Ninguna; producto en investigación. \\
\hline Fenofibrato & 1 & No & Ninguna; producto en investigación. \\
\hline Hidroxicloroquina $^{\mathrm{b}}$ & 2 & Sí & $\begin{array}{l}\text { Paludismo, amebiasis extraintestinal, lupus eritemato- } \\
\text { so y artritis reumatoide. }\end{array}$ \\
\hline IFX-1 & 1 & No & Ninguna; producto en investigación. \\
\hline Interferón ${ }^{\mathrm{b}}$ & 1 & Sí & $\begin{array}{l}\text { Artritis reumatoide, arteritis de células gigantes, } \\
\text { artritis idiopática juvenil y síndrome de liberación de } \\
\text { citocinas. }\end{array}$ \\
\hline Ivermectina & 1 & Sí & $\begin{array}{l}\text { Antihelmíntico: tratamiento de elección para Stron- } \\
\text { gyloides stercolaris, Filariasis }\end{array}$ \\
\hline Lactoferrina & 1 & Sí & Deficiencias de hierro \\
\hline Lopinavir/ritonavir ${ }^{\mathrm{b}}$ & 1 & Sí & VIH-1. \\
\hline Losmapimod & 1 & No & Ninguna; producto en investigación. \\
\hline LY3819253 (mAB) & 1 & No & Ninguna; producto en investigación. \\
\hline Mavrilimumab & 1 & No & Ninguna; producto en investigación. \\
\hline OT-101 & 1 & No & Ninguna; producto en investigación. \\
\hline Otilimab & 1 & No & Ninguna; producto en investigación. \\
\hline Plasma convaleciente & 4 & No & En investigación. \\
\hline Remdesivir & 1 & No & Ninguna; producto en investigación. \\
\hline Ruxolitinib & 1 & No & Ninguna; producto en investigación. \\
\hline TL-895 & 1 & No & Ninguna; producto en investigación. \\
\hline Tocilizumab & 1 & Sí & $\begin{array}{l}\text { Hepatitis } \mathrm{B} \text {, hepatitis } \mathrm{C} \text {, leucemia, mieloma múltiple, } \\
\text { linfoma folicular, tumor carcinoide y melanoma } \\
\text { maligno. }\end{array}$ \\
\hline Vacuna & 4 & No & Ninguna; producto en investigación. \\
\hline VIR-7831 & 1 & No & Ninguna; producto en investigación. \\
\hline
\end{tabular}

EC: ensayo clínico

a Según consulta a fichas técnica de especialidades farmacéuticas de la Dirección General de Medicamentos Insumos y Drogas.

${ }^{\mathrm{b}}$ Un EC que evaluaba estos productos en investigación determinó la ausencia de beneficio (futilidad)

sido la excepción. A los 30 días de declarada la emergencia sanitaria a nivel nacional, se emitió la primera norma para promover la conducción de EC en COVID-19. Los ajustes regulatorios están orientados a agilizar la actuación de las partes involucradas en la autorización de los EC, sin que se vea comprometida la evaluación rigurosa de sus aspectos administrativos, científicos y éticos, que garantizan la protección de los participantes y la validez de los datos generados. Los tiempos de revisión ética y autorización de los EC de la COVID-19 se han reducido; y se ha promovido un mayor seguimiento a su ejecución pues se ha triplicado la proporción de EC supervisados por el INS. Las acciones implementadas obligarán a revisar la regulación vigente, con miras a diseñar una más adecuada para la promoción de la investigación, particularmente de los EC, en el país.

Contribuciones de autoría: Todos los autores conceptualizaron el manuscrito, analizaron los datos, elaboraron el primer borrador del manuscrito, aprobaron la versión final del manuscrito y asumen la responsabilidad de su contenido.

Financiamiento: El estudio fue financiado por el Instituto Nacional de Salud. 
Conflicto de interés: PCL es coordinadora del área de evaluación de ensayos clínicos de la Oficina General de Investigación y Transferencia Tecnológica del Instituto Nacional de Salud. SC es consultora para temas de ética en investigación en la Oficina General de Investigación y Transferencia

\section{REFERENCIAS BIBLIOGRÁFICAS}

1. Gianola S, Jesus TS, Bargeri S, Castellini G. Characteristics of academic publications, preprints, and registered clinical trials on the COVID-19 pandemic. Mathes T, editor. PLOS ONE. 2020; 15(10):e0240123. doi:10.1371/journal.pone. 0240123

2. Lu Wang L, Lo K, Chandrasekhar Y, Reas R, Yang J, Eide D, et al. CORD19: The Covid-19 Open Research Dataset. arXiv:2004.10706v2.

3. Iserson KV. SARS-CoV-2 (COVID-19) Vaccine Development and Production: An Ethical Way Forward. Camb Q Healthc Ethics. 2021;30(1):5968. doi:10.1017/S096318012000047X

4. Olimid AP, Olimid D-A. Ethical review of patient safety and public health in EU clinical trials legislation: impact of COVID-19 pandemic. Rom J Morphol Embryol. 2020; 61(1):277-81. doi:10.47162/RJME.61.1.34

5. FDA Guidance on Conduct of Clinical Trials of Medical Products during COVID-19 Public Health Emergency. Guidance for Industry, Investigators, and Institutional Review Boards. March 2020. Updated on September 21, 2020 [citado el 14 de octubre del 2020]. Disponible en: https://www. fda.gov/regulatory-information/search-fda-guidance-documents/ fda-guidance-conduct-clinical-trials-medical-products-during-covid-19-public-health-emergency

6. Organización Panamericana de la Salud. Orientación ética sobre cuestiones planteadas por la pandemia del nuevo coronavirus (COVID-19). Programa Regional de Bioética. Departamento de Sistemas y Servicios de Salud., 2020. OPS/HSS/BIO/COVID-19/20-0008. [citado el 14 de octubre del 2020]. Disponible en: https://www.paho.org/es/documentos/orientacion-etica-sobre-cuestiones-planteadas-por-pandemia-nuevo-coronavirus-covid-19

7. World Health Organization. Council for International Organizations of Medical Sciences. International ethical guidelines for health-related research involving humans. Geneva: CIOMS; 2017 [citado el 8 de octubre del 2020]. Disponible en:https://cioms.ch/wp-content/uploads/2017/01/ WEB-CIOMS-EthicalGuidelines.pdf

8. Organización Panamericana de la Salud. Orientación y estrategias para agilizar la revisión y supervisión éticas de investigaciones relacionadas con COVID-19. Programa Regional de Bioética. Departmento de Sistemas y Servicios de Salud., 2020.OPS/HSS/BIO/Covid-19/20-0004. [citado el 8 de octubre del 2020]. Disponible en: https://www.paho.org/ es/documentos/orientacion-estrategias-para-agilizar-revision-supervision-eticas-investigaciones

9. Fernandez Lynch H, Dickert NW, Zettler PJ, Joffe S, Largent EA. Regulatory flexibility for COVID-19 research. J Law Biosci. 2020;7(1). doi:10.1093/jlb/lsaa057

10. Organización Panamericana de la Salud. Modelo y pautas operativas para la revisión y supervisión éticas de las investigaciones relacionadas con COVID-19. 2020 [citado el 19 de octubre de 2020]. Disponible en: https://iris.paho.org/bitstream/handle/10665.2/52087/OPSHSSBIOCOVID19200007_spa.pdf?sequence $=6$ \&isAllowed $=\mathrm{y}$

11. Resolución Jefatural Nº96-2020-J-OPE/INS. Conforman el Comité Nacional Transitorio de Ética en Investigación para la evaluación y supervisión ética de los Ensayos Clínicos de la enfermedad COVID-19. 2020. [citado el 8 de octubre del 2020]. Disponible en: https://busquedas. elperuano.pe/normaslegales/conforman-el-comite-nacional-transitorio-de-etica-en-investi-resolucion-jefatural-no-096-2020-j-opeins-1865513-1/

12. Resolución Jefatural $\mathrm{N}^{\circ} 097-2020-\mathrm{J}-\mathrm{OPE}-\mathrm{INS}$. Procedimiento para la revisión Ética de Ensayos clínicos de la Enfermedad COVID-19. 2020. [citado el 8 de octubre del 2020]. Disponible en: https://ensayosclinicos-repec. ins.gob.pe/images/RJ_COVID-19/RJ_097-2020-J-OPE-INS_-_PROCEDIMIENTO_CNTEI-COVID19.pdf
Tecnológica del Instituto Nacional de Salud. FR se desempeña como Director Ejecutivo de la Oficina de Transferencia Tecnológica y Capacitación del Instituto Nacional de Salud. Además, es el Editor Científico de la Revista Peruana de Medicina Experimental y Salud Pública.
13. Resolución Jefatural Nº98-2020-J-OPE/INS. Procedimiento de autorización para la realización de los Ensayos Clínicos de la enfermedad COVID-19. 2020. [citado el 8 de octubre del 2020]. Disponible en: https://ensayosclinicos-repec.ins.gob.pe/images/RJ_COVID-19/ RJ_098-2020-J-OPE-INS_-_PROCEDIMIENTO_AUTORIZACI\%C3\%93N_EC_I-COVID19.pdf

14. Organización Panamericana de la Salud. Consideraciones para la supervisión regulatoria de los ensayos clínicos en la pandemia de COVID-19. [Internet]. 2020. [citado el 8 de octubre del 2020] Disponible en: https:// iris.paho.org/handle/10665.2/52303

15. Alarcón Ruiz C, Roque-Roque J, Heredia P, Gómez-Briceno A, Quispe AM. Twenty-two years' experience registering trials in a low-middle income country: The Peruvian Clinical Trial Registry. J Evid Based Med. 2019; 1-7. doi:https://doi.org/10.1111/jebm.12354

16. Lanata De las Casas C, Gotuzzo HE. Estrategias y situación actual de la carrera para el desarrollo de vacunas eficaces y seguras para controlar la pandemia causada por el SARS-COV-2. Rev Peru Med Exp Salud Publica. 2020;37(3):401-2. https://doi.org/10.17843/rpmesp.2020.373.6522.

17. Lumpkin MM, Lim JCW. Pandemic Best Regulatory Practices: An Urgent Need in the COVID-19 Pandemic. Clin Pharmacol Ther. 2020; 108(4):703-5. doi:10.1002/cpt.1932

18. Perez T, Perez RL, Roman J. Conducting Clinical Research in the Era of Covid-19. Am J Med Sci. 2020; 360(3):213-5. doi:10.1016/j.amjms.2020.06.011

19. Hrabovszki G. Guidance for medicine developers and other stakeholders on COVID-19 [Internet]. European Medicines Agency. 2020 [citado el 17 de octubre de 2020]. Disponible en: https:// www.ema.europa.eu/en/human-regulatory/overview/public-health-threats/coronavirus-disease-covid-19/guidance-medicine-developers-other-stakeholders-covid-19

20. U.S. Food and Drug Administration. Coronavirus Treatment Acceleration Program (CTAP). FDA [Internet]. 2020 [citado el 17 de octubre de 2020]. Disponible en: https://www.fda.gov/drugs/coronavirus-covid-19-drugs/ coronavirus-treatment-acceleration-program-ctap

21. Agência Nacional de Vigilância Sanitária - Anvisa. Legislação COVID-19 [Internet]. [citado el 20 de octubre de 2020]. Disponible en: http://www. planalto.gov.br/ccivil_03/Portaria/quadro_portaria.htm

22. Instituto Nacional de Salud (Perú). Regulación de ensayos clínicos en la enfermedad COVID-19 [Internet]. [citado el 20 de octubre de 2020]. Disponible en: https://ensayosclinicos-repec.ins.gob.pe/regulacion/ normatividad-vigente/314-investigaciones-con-covid-19.

23. Resolución Ministerial N³39-2020-MINSA. Directiva Sanitaria $\mathrm{N}^{\circ}$ 103-MINSA/2020/DIGDOT. Directiva Sanitaria que establece los lineamientos técnicos para la obtención de plasma de donantes convalecientes de la enfermedad del Coronavirus (COVID-19). [citado el 15 de octubre del 2020]. Disponible en: https://cdn. www.gob.pe/uploads/document/file/753347/RM_339-2020MINSA.PDF

24. Minaya G, Fuentes D, Obregon C, Ayala Quintanilla B, Yagui Moscoso M. Características de los ensayos clínicos autorizados en el Perú, 1995-2012. Rev Peru Med Exp Salud Publica. 2012; 29(4):431-6.

25. Dyer O. Covid-19: Remdesivir has little or no impact on survival, WHO trial shows. BMJ [Internet]. 2020 [citado el 19 de octubre de 2020]; 371. doi:10.1136/bmj.m4057

26. Mahase E. Covid-19: Oxford researchers halt vaccine trial while adverse reaction is investigated. BMJ [Internet]. 2020 [citado el 19 de octubre de 2020]; 370. doi:10.1136/bmj.m3525 